\title{
A NECESSARY AND SUFFICIENT CONDITION FOR LOWER SEMICONTINUITY ${ }^{1}$
}

\author{
BY LAMBERTO CESARI
}

Communicated by M. Protter, August 1, 1973

In the present paper we state a few lower semicontinuity and lower closure theorems we have recently proved in connection with weak convergence. In particular, for simple integrals of the calculus of variations and weak convergence, we prove here that the convexity of the integrand with respect to the derivatives is a necessary and sufficient condition for lower semicontinuity.

1. Simple integrals of the calculus of variations. In this section we consider only functionals of the form

$$
I[x]=\int_{a}^{b} f_{0}\left(t, x(t), x^{\prime}(t)\right) d t, \quad\left({ }^{\prime}\right)=d / d t .
$$

We denote by $A$ a given closed subset of the $t x$-space $E^{n+1}\left(t \in E^{1}\right.$, $\left.x=\left(x^{1}, \cdots, x^{n}\right) \in E^{n}\right)$, and for the sake of simplicity we assume in this section that $A=\operatorname{cl}($ int $A)$. Here, $f_{\circ}(t, x, u)$ is a given function, $f_{\circ}$ : $A \times E^{n} \rightarrow E^{1}$, which again in this section will be assumed to be continuous on $A \times E^{n}$. Then the functional $I: \tau \rightarrow[-\infty,+\infty]$ is defined in the class $\tau$ of all absolutely continuous functions $x(t)=\left(x^{1}, \cdots, x^{n}\right), a \leqq t \leqq b$, whose graph lies in $A$, and for which the measurable function $f_{0}(t, x(t)$, $\left.x^{\prime}(t)\right), a \leqq t \leqq b$, has a Lebesgue integral on $[a, b]$ (finite, or $+\infty$, or $-\infty$ ).

We just note here that $\mathscr{F}: x \rightarrow f_{0}\left(\cdot, x(\cdot), x^{\prime}(\cdot)\right)$ is a Carathéodory, or Nemitskii operator [7].

For a sequence $x_{k}(t)=\left(x_{k}^{1}, \cdots, x_{k}^{n}\right), a \leqq t \leqq b, k=1,2, \cdots$, of absolute continuous functions we consider here the mode of convergence defined by $(m) x_{k}^{\prime}$ converges weakly in $L_{1}[a, b]$ and $x_{k}$ converges uniformly in $[a, b]$. This mode of convergence is justified by the simple remark that, if $x_{k}^{\prime}$ converges weakly in $L_{1}[a, b]$ and, for at least one $\bar{t} \in[a, b], x_{k}(\bar{t})$ converges (in $E^{n}$ ), then $x_{k}$ converges uniformly in $[a, b]$. This remark is a simple consequence of the Dunford-Pettis theorem [6].

The interest of this mode of convergence lies in the fact that a necessary and sufficient condition for the lower semicontinuity of $I[x]$ at every

\footnotetext{
AMS (MOS) subject classifications (1970). Primary 49A25, 49A50; Secondary 49A05.

1 This research was partially supported by AFOSR Research Project 71-2122 at the University of Michigan. 
element $x \in \tau$ (with respect to the mode $(m)$ of convergence) is that $f_{\circ}(t, x, u)$ be convex with respect to $u$ for every $(t, x) \in A$. The necessity is proved by the same Tonelli classical argument (for the uniform convergence only of the functions $x_{k}$ ) (see, e.g. [1]). The sufficiency is proved by the following statement.

(1.i) (CESARI [2]) (A LOWER SEMICONTINUITY THEOREM FOR SIMPLE INTEGRALS). If $A$ is closed, $f_{\circ}$ continuous on $A \times E^{n}$ and convex in $u$ for every $(t, x) \in A$, if $x(t), x_{k}(t), a \leqq t \leqq b, k=1,2, \cdots$, are absolutely continuous functions with graphs in $A$, such that $x_{k} \rightarrow x$ uniformly in $[a, b]$ and $x_{k}^{\prime} \rightarrow x^{\prime}$ weakly in $\left(L_{1}[a, b]\right)^{n}$, then $I[x] \leqq \lim _{\inf _{k \rightarrow \infty}} I\left[x_{k}\right]$, and each of these integrals necessarily is either finite, or $+\infty$.

This statement is actually a corollary of the more general statements below.

2. Multiple integrals of the calculus of variations. In this section we consider functionals of the form

$$
I[x]=\int_{G} f_{\circ}(t, x(t), \nabla x(t)) d t,
$$

where $G$ is a bounded domain of the $t$-space $E^{v}, v \geqq 1, t=\left(t^{1}, \cdots, t^{v}\right)$, $A$ is a closed subset of the $t x$-space $E^{v+n}, x=\left(x^{1}, \cdots, x^{n}\right)$, whose projection on the $t$-space is $\operatorname{cl~} G$, and $f_{0}(t, x, u)$ is a given function $f_{0}$ : $A \times E^{v n} \rightarrow E^{1}$, which again in this section will be assumed to be continuous on $A \times E^{v n}$. We think of $I[x]$ as a functional defined in the class $\tau$ of all functions $x(t), t \in G$, of the Sobolev space $\left(W_{1}^{1}(G)\right)^{n}$ whose graph lies in $A$ and for which $f_{0}(\cdot, x(\cdot), \nabla x(\cdot))$ has a Lebesgue integral (finite, or $+\infty$, or $-\infty)$. The following statement is a lower semicontinuity theorem for the integral (2) in connection with the usual weak convergence in the Sobolev space $\left(W_{1}^{1}(G)\right)^{n}$ (that is, $\nabla x_{k}$ converges weakly in $\left(L_{1}(G)\right)^{v n}$ and $x_{k}$ converges strongly in $\left.\left(L_{1}(G)\right)^{n}\right)$.

(2.i) (CESARI [2]) (A LOWER SEMICONTINUITY THEOREM FOR MULTIPLE INTEGRALS). Let $G$ be a bounded domain, let $A$ be closed, and $f_{\circ}(t, x, u)$ continuous in $A \times E^{v n}$, bounded in the subset $[(t, x, u)|(t, x) \in A| u \mid, \leqq 1]$, and convex in $u$ for every $(t, x) \in A$. If $x(t), x_{k}(t), t \in G, k=1,2, \cdots$, are given functions, $x, x_{k} \in\left(W_{1}^{1}(G)\right)^{n}$, with graphs in $A$, and $x_{k} \rightarrow x$ weakly in $\left(W_{1}^{1}(G)\right)^{n}$, then $I[x] \leqq \lim \inf _{k \rightarrow \infty} I\left[x_{k}\right]$, and each of these integrals necessarily is either finite, or $+\infty$.

This statement (2.i) is a corollary of the following Theorems (3.i) and (4.i) in a more general setting. The boundedness condition of $f_{\circ}$ on the set $[(t, x, u)|(t, x) \in A| u \mid, \leqq 1]$ is a very mild one since it is certainly 
satisfied whenever $A$ is compact (and $f_{\circ}$ is continuous). We proved in [2], [3] and [5] also other lower semicontinuity theorems for integrands $f_{0}$ not necessarily continuous but satisfying suitable analytical conditions (Lipschitz-type with respect to $x$, and growth type as $|u| \rightarrow \infty$ ). Finally, we refer here to C. B. Morrey's book [8] for other semicontinuity theorems under different modes of convergence.

3. Integrals $I[x, u]$ and Carathéodory continuity condition (C). In this section we consider functionals of the form

$$
I[x, u]=\int_{G} f_{\circ}(t, x(t), u(t)) d t,
$$

where $G$ is any measurable subset of finite measure of the $t$-space $E^{v}$, $t=\left(t^{1}, \cdots, t^{\nu}\right), v \geqq 1, A$ is any subset of the $t x$-space $E^{v+n}, x=\left(x^{1}, \cdots, x^{n}\right)$, whose projection on the $t$-space is $G, M$ is the subset of the $t x u$-space $E^{v+n+m}, u=\left(u^{1}, \cdots, u^{m}\right)$, defined by $M=A \times E^{m}$, and $f_{0}(t, x, u)$ is a given function $f_{0}: M \rightarrow E^{1}$. For every $t \in G$ we denote by $A(t)$ the set $A(t)=\left[x \in E^{n} \mid(t, x) \in A\right]$. We denote by $|H|$ the Lebesgue measure of measurable subsets $H$ of $E^{v}$.

We shall say that condition (C) holds provided, for every $\varepsilon>0$, there is a compact subset $K$ of $G$ with $|G-K|<\varepsilon$ such that the set $A_{K}=[(t, x) \in$ $A \mid t \in K]$ is closed, and $f_{0}(t, x, u)$ is continuous on $M_{K}=A_{K} \times E^{m}$.

If $A$ and $M$ are closed, and $f_{\circ}$ is continuous in $(x, u)$ for every $t$, and measurable in $t$ for every $(x, u)$, then condition (C) certainly holds. If condition (C) holds, then for almost all $\bar{t} \in G$ the set $A(\bar{t})=\left[x \in E^{n} \mid(\bar{t}, x) \in A\right]$ is closed and $f_{0}(\bar{t}, x, u)$ is continuous on the set $M(\bar{t})=A(\bar{t}) \times E^{m}$.

Under condition (C), then for every pair of measurable functions $x(t), u(t), t \in G$, with $x(t) \in A(t)$ for $t \in G$ (a.e.), the function $f_{\circ}(\cdot, x(\cdot)$, $u(\cdot))$ is measurable in $G$. We think of the functional $I$ as defined on all such pairs $x, u$, for which $f_{0}(\cdot, x(\cdot), u(\cdot))$ has a Lebesgue integral (finite, or $+\infty$, or $-\infty$ ).

(3.i) (CESARI [2]) (A LOWER SEMICONTINUITY THEOREM FOR $I[x, u]$ ). Let $G$ be measurable with finite measure, let us assume that condition (C) holds, that $f_{0}(t, x, u)$ is bounded on the subset $[(t, x, u)|(t, x) \in A| u \mid, \leqq 1]$, and that $f_{0}(t, x, u)$ is convex in $u$ for every $(t, x) \in A$. Let $x(t), x_{k}(t), u(t)$, $u_{k}(t), t \in G, k=1,2, \cdots$, be measurable functions on $G, x, x_{k}: G \rightarrow E^{n}$, $u, u_{k} \in\left(L_{1}(G)\right)^{m}$, such that $x_{k}(t) \in A(t), t \in G($ a.e. $), x_{k} \rightarrow x$ in measure, and $u_{k} \rightarrow u$ weakly in $\left(L_{1}(G)\right)^{m}$ as $k \rightarrow \infty$. Then $x(t) \in A(t), t \in G$ (a.e.), and $I[x, u] \leqq \lim \inf _{k \rightarrow \infty} I\left[x_{k}, u_{k}\right]$, where each of these integrals is necessarily finite, or $+\infty$.

For this and other lower semicontinuity theorems we refer to [2] and [3]. 
4. Lower closure theorems of optimal control theory. In this section we consider functionals of the form

$$
I[x, u]=\int_{G} f_{\circ}(t, x(t), u(t)) d t
$$

under constraints of the form

$$
\begin{aligned}
& \xi(t)=f(t, x(t), u(t)), \quad t \in G \quad \text { (a.e.), } \\
& x(t) \in A(t), \quad u(t) \in U(t, x(t)), \quad t \in G \quad \text { (a.e.). }
\end{aligned}
$$

Here $G$ is any measurable subset of finite measure of the $t$-space $E^{v}$, $t=\left(t^{1}, \cdots, t^{v}\right), v \geqq 1$. For every $t \in G$ we denote by $A(t)$ a given subset of the $x$-space $E^{n}, x=\left(x^{1}, \cdots, x^{n}\right)$, and then $A$ is the set $A=[(t, x) \mid t \in G$, $x \in A(t)] \subset E^{v+n}$. For every $(t, x) \in A$ we denote by $U(t, x)$ a given subset of the $u$-space $E^{m}, u=\left(u^{1}, \cdots, u^{m}\right)$, and then $M$ is the set $M=[(t, x, u)$ $(t, x) \in A ; u \in U(t, x)] \subset E^{v+n+m}$, and $f_{\circ}(t, x, u), f(t, x, u)=\left(f_{1}, \cdots, f_{r}\right)$ are given functions defined on $M$. We shall assume that condition (C) holds for both $f_{\circ}$ and $f$.

We shall consider here triples of measurable functions $\xi(t), x(t), u(t)$, $t \in G$, with values in $E^{r}, E^{n}, E^{m}$ respectively for which (5), (6) hold a.e. in $G$, and for which the measurable function $f_{0}(\cdot, x(\cdot), u(\cdot))$ has a Lebesgue integral (finite, or $+\infty$, or $-\infty$ ). In all applications there are functional relations between the functions $x$ and $\xi$, which are not relevant in the present context.

For every $(t, x) \in A$ we shall consider the set

$$
\widetilde{Q} \quad x)=\left[\left(z^{\circ}, z\right) \mid z^{\circ} \geqq f_{\circ}(t, x, u), z=f(t, x, u), u \in U(t, x)\right] \subset E^{r+1},
$$

and its projection

$$
Q(t, x)=f(t, x, U(t, x)) \subset E^{r}
$$

on the $z$-space $E^{r}$. For every $z \in Q(t, x)$ we can now define

$$
T(z ; t, x)=\operatorname{Inf}\left[z^{\circ} \mid\left(z^{\circ}, z\right) \in \widetilde{Q}(t, x)\right], \quad-\infty \leqq T(z ; t, x)<+\infty,
$$

that is, the infimum of all $z^{\circ}=f_{\circ}(t, x, u)$ for $u \in U(t, x)$ with $z=f(t, x, u)$. We have proved elsewhere that, if $\widetilde{Q}(t, x)$ is convex, so is $Q(t, x)$ and then, either $T(z ; t, x)=-\infty$ for all $z \in R$ int $Q(t, x)$, or $T(z ; t, x)>-\infty$ for all $z \in Q(t, x)$ and $T(z ; t, x)$ is a convex function of $z$ on the convex set $Q(t, x)$. Here $R$ int $Q(t, x)$ denotes the set of all points $z$ which are interior to $Q(t, x)$ with respect to the hyperspace $R$ of minimum dimension containing $Q(t, x)$, say, int $Q \subset R$ int $Q \subset Q \subset R \subset E^{r}$.

For every $\bar{t} \in G, \bar{x} \in A(\bar{t})$, and $\varepsilon<0$ let $N_{\delta, t}(\bar{x})$ denote the set of all $x \in A(\bar{t})$ with $|x-\bar{x}| \leqq \delta$. For every $\bar{t} \in G$ we may now consider the sets 
$\widetilde{\widetilde{Q}}(t, x), x \in A(\bar{t})$ (or analogous ones). We shall say that these sets have Kuratowski's upper semicontinuity property (property $(\mathrm{K})$ ) with respect to $x$ at a point $\bar{x} \in A(\bar{t})$ provided

$$
\widetilde{Q}(\bar{t}, \bar{x})=\bigcap_{\delta} \mathrm{cl} \bigcup_{x \in N_{\delta, t}(\bar{x})} \widetilde{Q}(\bar{t}, x) ;
$$

and that they have property $(\mathrm{Q})$ with respect to $x$ at $\bar{x}$ provided

$$
\widetilde{Q}(\bar{t}, \bar{x})=\bigcap_{\delta} \operatorname{clco} \bigcup_{x \in N_{\delta},(\bar{x})} \widetilde{Q}(t, x) .
$$

We shall say that for any given $\bar{t} \in G$ the sets $\widetilde{Q}(t, x)$ have property (K) or $(\mathrm{Q})$ with respect to $x$ in $A(\bar{t})$ if they have the corresponding property above at every $\bar{x} \in A(\bar{t})$. For any $N>0$ we denote by $V(0, N)$ the set $V(0, N)=[z|| z \mid \leqq N] \subset E^{r}$.

(4.i) (CeSARI [2]) (A LOWER ClOSURE THEOREM). Let $\mathrm{G}$ be measurable with finite measure, let us assume that condition (C) holds for both $f_{0}$ and $f$, that for almost all $t \in G$ and all $x \in A(t)$ the sets $\widetilde{Q}(t, x)$ are closed and convex. Let us assume also that $(\alpha)$ there is a measurable bounded function $p(t), t \in G,|p(t)| \leqq \sigma, p: G \rightarrow E^{r}$, such that $p(t) \in Q(t, x)$ for almost all $t \in G$ and all $x \in A(t) ;(\beta)$ there is a constant $c>0$ such that $|T(z ; t, x)| \leqq c$ for almost all $t \in G$, all $x \in A(t)$, and $z \in Q(t, x) \cap V(0,2 \sigma) ;(\gamma)$ for every $N>2 \sigma$ and almost all $t \in G$ the sets $\widetilde{Q}(t, x) \cap\left(E^{1} \times V(0, N)\right)$ satisfy property $(\mathrm{K})$ with respect to $x$ in $A(t)$. If $\xi(t), x(t), \eta_{k}(t), \xi_{k}(t), x_{k}(t), u_{k}(t)$, $t \in G, k=1,2, \cdots$, are measurable functions, $\xi, \xi_{k} \in\left(L_{1}(G)\right)^{r}, \eta_{k} \in L_{1}(G)$, if $x_{k}(t) \in A(t), u_{k}(t) \in U\left(t, x_{k}(t)\right), \eta_{k}(t)=f_{0}\left(t, x_{k}(t), u_{k}(t)\right), \xi_{k}(t)=f\left(t, x_{k}(t)\right.$, $\left.u_{k}(t)\right), t \in G, k=1,2, \cdots, \xi_{k} \rightarrow \xi$ weakly in $\left(L_{1}(G)\right)^{r}, x_{k}(t) \rightarrow x(t)$ in measure in $G$ as $k \rightarrow \infty$, and $i=\lim _{\inf _{k}} \int_{G} \eta_{k}(t) d t<+\infty$, then $i$ is finite and there is some measurable function $u(t), t \in G$, such that $x(t) \in A(t), u(t) \in$ $U(t, x(t)), \quad \xi(t)=f(t, x(t), u(t)), t \in G($ a.e. $)$, and, for $\eta(t)=f_{0}(t, x(t)$, $u(t)), t \in G$, we also have $\eta \in L_{1}(G)$, and $\int_{G} \eta(t) d t \leqq i$.

Note that, for every $N$, the sets $\widetilde{\widetilde{Q}}(t, x) \cap\left(E^{1} \times V(0, N)\right)$ are contained in the fixed set $E^{1} \times V(0, N)$, and are closed and convex. As proved elsewhere, the required condition $(\mathrm{K})$ with respect to $x$ for the sets $\widetilde{Q}(t, x) \cap$ $\left(E^{1} \times V(0, N)\right)$ is certainly satisfied if we know that $|f(t, x, u)| \rightarrow+\infty$ as $|u| \rightarrow+\infty$ uniformly with respect to $(t, x)$ in $A$.

This theorem (4.i) contains the previous statements of $\S \S 1,2,3$ as particular cases, and was recently proved in [2] on the basis of previous work in [4]. We proved also in [4] (and presented in [3]) other lower closure theorems where conditions $(\alpha),(\beta),(\gamma)$ are replaced by analytic conditions on $f_{\circ}$ and $f$ as Lipschitz type conditions with respect to $x$, or growth type conditions on $f_{\circ}$ and $f$ as $|u| \rightarrow \infty$. 


\section{REFERENCES}

1. L. Cesari, Problems of optimization (to appear).

2. - Lower semicontinuity and lower closure theorems without seminormality conditions, Ann. Mat. Pura Appl. 98 (1974), 381-397.

3. — Closure theorems for orientor fields, Bull. Amer. Math. Soc. 79 (1973), $682-687$.

4. - Closure theorems for orientor fields and weak convergence, Arch. Rational Mech. Anal. (to appear).

5. L. Cesari and M. B. Suryanarayana, Closure theorems without seminormality conditions, J. Optimization Theory Appl. (to appear).

6. R. E. Edwards, Functional analysis. Theory and applications, Holt, Rinehart and Winston, New York, 1965, p. 271 (4.21.1). MR 36 \#4308.

7. M. A. Krasnosel'skiŭ, Topological methods in the theory of nonlinear integral equations, GITTL, Moscow, 1956; English transl., Macmillan, New York, 1964, pp. 20-32. MR 20 \#3464; 28 \#2414.

8. C. B. Morrey, Multiple integrals in the calculus of variations, Die Grundlehren der Math. Wissenschaften, Band 130, Springer-Verlag, New York, 1966.

Department of Mathematics, University of Michigan, Ann Arbor, Michigan 48104 